\title{
tic\&société
}

Vol. $1, n^{\circ} 1 \mid 2007$

De TIS à tic\&société : dix ans après

\section{De Marshall McLuhan à Harold Innis ou du village global à l'empire mondial}

\section{Gaëtan Tremblay}

\section{(2) OpenEdition}

Journals

Édition électronique

URL : http://journals.openedition.org/ticetsociete/222

DOI : 10.4000/ticetsociete.222

Éditeur

Association ARTIC

\section{Référence électronique}

Gaëtan Tremblay, « De Marshall McLuhan à Harold Innis ou du village global à l'empire mondial »,

tic\&société [En ligne], Vol. 1, n¹ | 2007, mis en ligne le 15 février 2008, consulté le 10 décembre 2020

URL : http://journals.openedition.org/ticetsociete/222 ; DOI : https://doi.org/10.4000/ticetsociete.222 


\title{
De Marshall McLuhan à Harold Innis ou du village global à l'empire mondial'
}

\author{
Gaëtan TREMBLAY, Professeur \\ Adresse postale : \\ Université du Québec à Montréal \\ Case postale 8888, succursale Centre-ville \\ Montréal (Québec) H3C 3P8 \\ CANADA \\ Adresse courriel : \\ tremblay.gaetan@uqam.ca
}

Gaëtan TREMBLAY est professeur à l'Université du Québec à Montréal (UQAM) depuis 1974 (d'abord au département des Communications puis à l'École des médias) et directeur du Centre d'études et de recherches sur le Brésil (CERB) depuis 2004. Co-fondateur du Groupe de recherche interdisciplinaire sur la communication, l'information et la société (GRICIS), il a dirigé une quinzaine d'ouvrages collectifs et publié plus d'une centaine de contributions à des revues et livres sur les communications, les industries culturelles et médiatiques.

\footnotetext{
${ }^{1}$ Un texte sur le même thème a été présenté sous forme de communication lors d'un colloque organisé par l'Université catholique du Rio Grande do Sur, les 25-26 août 2003, et publié par la suite en portugais dans la Revista FAMECOS: mídia, cultura e tecnologia, Porto Alegre, no 22, dezembro 2003, p. 13-22. Cette version est en revanche inédite.
} 


\section{Gaëtan TREMBLAY}

\section{Résumé}

L'auteur présente ici une lecture personnelle de la contribution aux études en communication de deux pionniers canadiens, Marshall McLuhan et Harold A. Innis, le premier toujours considéré par plusieurs comme le gourou des technologies de l'information et de la communication, le second encore trop souvent ignoré de la grande majorité des chercheurs. À rebours des interprétations habituelles qui en soulignent les affinités, il met en évidence leurs différences. Refusant d'emblée leur déterminisme technologique, il propose une synthèse compréhensive et critique de leurs cadres d'analyse et de leurs démarches méthodologiques, cherchant à évaluer l'influence de l'un et de l'autre dans son cheminement personnel, et à retracer les avancées et les dérives auxquelles ils ont contribué dans l'évolution de la recherche en communication. Le titre condense le point de vue de l'auteur : il faut remonter de McLuhan à Innis, passer de la grille de lecture qu'inspire la métaphore du village global à celle qu'appelle l'expansion de l'empire.

Mots-clés : . Innis, McLuhan, communication, civilisation, déterminisme technologique, empire, village global

\section{Abstract}

The author presents here a personal reading of two pioneering Canadians' contribution to communication studies, Marshall McLuhan and Harold A. Innis, the former still considered by many as information and communication technologies guru, the latter too often ignored by most researchers. Running counter the general trend stressing their affiliations, he highlights their differences. Rejecting their techological determinism standpoint, he proposes a comprehensive and critical summary of their analytical frameworks and methodologies, seeking to assess the influence one and the other had on his own perspective, and tracing the progresses and the drifts they contributed to in the evolution of communication research. The author condensed his viewpoint in the title: we should go back from McLuhan to Innis, from a framework inspired by the global village metaphor to the one called by the empire expansion.

Keywords: Innis, McLuhan, communication, civilisation, technological determinism, empire, global village .

\section{Resumen}

El autor presenta una lectura personal de la contribución de dos pioneros canadienses, Marshall McLuhan y Harold A. Innis, a los estudios comunicacionales. El primero ha sido considerado por muchos como el gurú de las tecnologías de la información y de la comunicación, mientras que el 
De Marshall McLuhan à Harold Innis
ou du village global à l'empire mondial

segundo continúa siendo ignorado por la mayoría de los investigadores. Al contrario de las interpretaciones usuales que subrayan sus afinidades, este artículo destaca sus diferencias. Rechazando de plano su determinismo tecnológico, se propone una síntesis comprehensiva, a la vez que crítica, de sus marcos de análisis y de sus aproximaciones metodológicas, tratando de evaluar la influencia de estas y aquellos en su pensamiento. A la vez se identifican sus contribuciones a los avances y derivas en la evolución de la investigación en comunicación. El punto de vista del autor se condensa en el título : resulta necesario remontar de McLuhan a Innis, y pasar de un planteamiento basado en la metáfora de la aldea global a otro basado en la expansión del imperio.

Palabras-clave: Innis, McLuhan, comunicación, civilización, determinismo tecnológico, imperio, aldea global. 


\section{Gaëtan TREMBLAY}

Marshall McLuhan est l'auteur canadien le plus connu et le plus cité dans le monde. Cette notoriété ne s'accompagne malheureusement pas d'une connaissance approfondie de son œuvre. Pour l'étudiant en communication, cette dernière se limite habituellement à une compréhension approximative, voire erronée, de la métaphore du village global et de l'aphorisme célèbre « le médium, c'est le message ». Pour l'analyste critique, McLuhan ne représente souvent que le plus illustre apologiste du déterminisme technologique et, de ce fait, ne mérite guère plus ample considération.

Harold A. Innis est moins connu à l'extérieur du Canada. Ses livres n'ont pas été traduits en plusieurs langues comme ceux de McLuhan et son influence, à quelques exceptions près, n'a que peu débordé les frontières canadiennes. Seuls quelques savants, parmi lesquels on compte l'illustre communicologue américain James Carey, lui ont prêté une studieuse attention et ont trouvé dans ses travaux une féconde inspiration. En revanche, il a laissé un héritage plus marquant que McLuhan dans les milieux académiques canadiens qui se consacrent à l'étude des phénomènes de communication, en particulier ceux qui se réclament d'une approche appartenant à la galaxie de l'économie politique.

Mon enseignement m'a amené à lire à plusieurs reprises les principaux ouvrages d'Innis et de McLuhan. Je ne prétends pas pour autant être exégète ou spécialiste ni de l'un ni de l'autre de ces auteurs. Mais l'un comme l'autre ayant exercé sur moi une profonde influence à deux moments différents de ma carrière, je suis heureux de l'occasion qui m'est offerte de reconnaître publiquement ma dette et de procéder à une évaluation critique de ce que j'estime être leur contribution aux sciences de la communication.

Lorsqu'on s'intéresse aux rapports entre les techniques de communication et l'organisation sociale, économique et politique; à la culture au sens large, c'està-dire aux modes de penser, de sentir, de connaître autant qu'aux œuvres littéraires et artistiques; à la production, à la distribution et à la consommation des produits culturels, informationnels et communicationnels; à l'impact des médias dans la vie des individus et des sociétés; la lecture des ouvrages d'Harold Innis et de Marshall McLuhan m'apparaît incontournable.

Je me propose de procéder, dans cet article, à une évaluation critique de l'apport de McLuhan et d'Innis aux sciences de la communication en exposant succinctement les principales idées et la méthode de travail de chacun. J'aborderai ensuite plus spécifiquement, comme l'annonce mon titre, les thèmes du village global et de l'empire, au cœur de l'actualité comme de l'œuvre des deux auteurs canadiens. Je conclurai sur quelques éléments de contexte qu'il 
De Marshall McLuhan à Harold Innis

ou du village global à l'empire mondial

faudrait approfondir pour bien apprécier l'originalité et l'importance des travaux d'Innis et de McLuhan.

\section{Deux pionniers des études en communication}

Les écrits de McLuhan sont postérieurs à ceux d'Innis et le gourou de Toronto a reconnu explicitement à quelques reprises le travail de pionnier de l'auteur d'Empire and Communication et de The Bias of Communication. Mais pour deux raisons, j'amorcerai mes réflexions à rebours de la séquence historique. Premièrement, parce que j'ai connu en premier McLuhan et, deuxièmement, parce que je pense que l'histoire récente nous invite à remonter de l'oeuvre de ce dernier vers celle d'Innis.

Les deux professeurs de Toronto ont en commun le même intérêt pour les médias de communication qu'ils ont placés au cœur de leurs travaux. On présente souvent Innis comme le précurseur de McLuhan, d'autant plus que ce dernier s'est réclamé du premier dont il a préfacé une réédition de Empire and Communication, en 1972, et rédigé l'introduction d'une réédition de The Bias of Communication, en 1977.

Mais, comme nous le verrons, l'analyse attentive de leurs travaux met à jour autant sinon plus de différences que de points communs. L'un a reçu une formation en sciences économiques, l'autre en études littéraires. Innis a fait une carrière universitaire classique alors que McLuhan en est toujours resté à la marge, plus toléré qu'intégré au monde académique. Innis a conduit ses recherches en appliquant rigoureusement les méthodes des sciences sociales. McLuhan a davantage emprunté à la façon de travailler des artistes. Le premier s'est intéressé davantage au sort des collectivités, le second à celui des individus. Innis est resté pessimiste jusqu'à la fin de sa vie quant à l'avenir des sociétés modernes, n'y trouvant guère réunies les conditions de reproduction d'un équilibre nécessaire à leur pérennité. McLuhan, en revanche, est passé d'un sentiment d'inquiétude devant les manifestations de la culture contemporaine, perceptible dans des ouvrages comme The Mechanical Bride et War and Peace in the Global Village, à un certain optimisme face aux possibilités de la civilisation de l'électricité, qu'il étale plus volontiers dans Understanding Media et The Medium is the Massage.

Si les deux auteurs canadiens sont restés fascinés par des sociétés du passé -McLuhan par le Moyen-Age et les sociétés tribales, Innis par le siècle d'or de la Grèce de Périclès- l'œuvre de chacun répond à des questions, à des 


\section{Gaëtan TREMBLAY}

préoccupations spécifiques, à un projet propre. Innis a essayé de déterminer les conditions de l'équilibre et de la pérennité des sociétés, des empires, des civilisations. McLuhan a tenté de comprendre les causes, le sens et la direction des mutations consécutives à l'invention de nouveaux médias et de leur impact sur la vie des hommes et des femmes modernes, ou post-modernes si l'on préfère.

\section{L'oracle McLuhan}

Mon premier contact avec l'œuvre de McLuhan remonte à l'été de 1967, mémorable pour tous les Québécois de ma génération parce que Montréal fut l'hôte cette année-là d'une Exposition universelle. Je venais de terminer le collège et j'allais entreprendre des études en sociologie à l'université Laval. La lecture de The Medium is the Massage, puis celle de The Gutenberg Galaxy et Undertanding Media, à travers l'excellente traduction française effectuée par le journaliste et éditeur québécois Jean Paré, allaient s'avérer déterminantes dans l'orientation de mon mémoire de maîtrise, et de mes recherches ultérieures, en sociologie de la communication. Je ne saurais dire si McLuhan est le principal facteur à l'origine de mon intérêt pour les communications mais je suis convaincu qu'il a tout au moins grandement contribué à le confirmer.

J'ai cependant toujours entretenu une relation ambivalente avec son œuvre. J'ai souvent été mal à l'aise avec ses imprécisions conceptuelles et ses affirmations à l'emporte-pièce. J'ai toujours été sceptique face à sa théorie de la perception et n'ai jamais adhéré à son déterminisme technologique. En revanche, j'ai fréquemment été stimulé par ses métaphores osées, ses rapprochements étonnants, ses interprétations originales d'œuvres littéraires et artistiques. Si j'avais à résumer en quelques mots mon rapport à McLuhan, je dirais que c'est un auteur avec lequel je suis très souvent en désaccord mais qui me provoque, m'interroge, me stimule, me fait réagir. Pour le paraphraser, je ne suis pas d'accord avec le contenu mais peu importe, c'est mon interaction avec le médium qui compte ! C'est précieux, un auteur qui fait réfléchir et j'ose penser que McLuhan serait plutôt flatté de ce rôle. Malheureusement, on se contente trop souvent de répéter comme des dogmes, souvent à contresens, ses plus célèbres formules.

McLuhan fut sans conteste l'un des premiers à attirer l'attention du public sur l'existence même des techniques de communication, sur leurs caractéristiques et leur mode de fonctionnement, plutôt que sur les seuls messages qu'ils véhiculent. Jusqu'aux années 1960, les chercheurs ne s'intéressaient guère qu'aux effets spécifiques de différents types de messages (à des fins de propagande ou de publicité) et le débat public sur les médias était obnubilé par 
De Marshall McLuhan à Harold Innis

ou du village global à l'empire mondial

la moralité des programmes. Les ouvrages de McLuhan sont venus à point nommé rappeler l'importance des techniques de diffusion et des réseaux de transmission. Le style qu'il a adopté, tout en formules lapidaires et flamboyantes, tout autant que le caractère dramatique et mystérieux de son message, ont fait de lui un oracle des communications.

\title{
2.1. Les médias révolutionnent le monde
}

Toute la pensée de McLuhan repose sur cette conviction profonde: les médias, qui définissent l'environnement de l'homme et de la société, bouleversent tous les aspects de la vie.

\begin{abstract}
The medium, or process, of our time -electric technology - is reshaping and restructuring patterns of social interdependence and every aspect of our personal life. It is forcing us to reconsider and reevaluate practically every thought, every action, and every institution formerly taken for granted. Everything is changing -you, your family, your neighbourhood, your education, your job, your government, your relation to "the others". And they're changing dramatically $^{2}$ (The Medium is the Massage, p. 8).
\end{abstract}

L'évolution des médias constitue le facteur explicatif principal, déterminant, de l'histoire humaine, que McLuhan divise en trois grandes périodes selon le média qui y domine : la civilisation de l'oralité, la civilisation de l'imprimerie (la galaxie Gutenberg) et la civilisation de l'électricité (la galaxie Marconi). On pourrait multiplier jusqu'à satiété les citations de passages qui ont fait de McLuhan, malgré ses occasionnelles dénégations, l'un des plus illustres hérauts du déterminisme technologique. Dans son œuvre, la société et l'individu sont modelés par les médias. Les facteurs sociaux, économiques, culturels ou

2 «Le médium, ou processus, de notre temps — la technologie électrique — remodèle et restructure les modes d'interdépendance sociale et tous les aspects de notre vie personnelle. II nous force à reconsidérer et réévaluer pratiquement chaque pensée et chaque action, chaque institution antérieurement prise pour acquise. Tout change vous, votre famille, votre voisin, votre éducation, votre emploi, votre gouvernement, votre relation « aux autres ». Et ils changent radicalement »(ma traduction). 


\section{Gaëtan TREMBLAY}

politiques, lorsqu'ils sont évoqués, n'ont jamais qu'une importance secondaire face à la surdétermination technique.

Certains passages des livres de McLuhan mettent beaucoup l'accent sur le caractère mécanique et industriel de la production médiatique: division et hiérarchisation des opérations, interchangeabilité des composantes, reproduction mécanique, linéarité de la pensée :

L'imprimerie a été la première mécanisation d'un art ancien et a conduit à la mécanisation ultérieure de tous les métiers manuels (La Galaxie Gutenberg, p. 70).

C'est le principe de fractionnement qui est l'essence même de la technologie mécanique, qui façonnait les structures de travail et d'association des humains. L'essence de la technologie de l'automation est tout à l'opposé. Elle est englobante et profondément décentralisatrice, alors que la machine était fractionnelle, centralisatrice et superficielle dans son façonnement des relations humaines (Pour comprendre les médias, p. 24).

Je ne saurais dire si McLuhan a lu les ouvrages de l'École de Francfort, en particulier ceux d'Adorno et d'Horkheimer. Mais s'il partage avec eux ce constat du caractère mécanique, industriel de la culture, il en fait une analyse complètement opposée. Pour les chercheurs de l'École de Francfort, ce sont les techniques modernes de communication, inventées au début du XXe siècle, en particulier la radio et le cinéma, qui sont responsables de cette industrialisation de la culture. Pour McLuhan, la mécanisation de la culture résulte plutôt de l'invention de l'imprimerie au XVe siècle. Pour Adorno et Horkheimer, I'homogénéisation consécutive à la reproduction industrielle menace la culture d'émancipation héritée des Lumières, qui s'incarne dans les grandes œuvres artistiques et littéraires des siècles passés diffusées en grande partie grâce à l'imprimerie. Pour McLuhan, les médias électriques et électroniques qui se succèdent depuis la seconde moitié du XIXe siècle autorisent un retour à la perception multi-sensorielle et à la pensée complexe et globale, caractéristiques de l'oralité, après les siècles de linéarité, de spécialisation, de hiérarchisation, de division et de sectarisme qui ont suivi l'invention, la diffusion et la domination de l'imprimé dans le monde civilisé :

L'homogénéisation des hommes et des objets deviendra le grand objectif de l'âge de Gutenberg, ainsi que la source d'une richesse et d'une puissance que n'ont connues aucune autre époque et aucune autre technologie (La Galaxie Gutenberg, pp. 188-189) 
De Marshall McLuhan à Harold Innis

ou du village global à l'empire mondial

L'homme unidimensionnel, pour McLuhan, ne résulte pas de l'expansion de la culture de masse et du système capitaliste, comme l'a écrit Herbert Marcuse (1969). Tout au contraire, il est fondamentalement, pour l'auteur de la Galaxie Gutenberg, le produit de la culture livresque rendue possible par l'invention de l'imprimerie. C'est un renversement de perspective complet par rapport aux théories de l'école de Francfort.

\subsection{Les médias, extensions du corps humain}

Ce constat de l'industrialisation de la culture et de la communication aurait pu conduire McLuhan à développer une perspective économique ou sociologique. II a plutôt choisi de lui donner un cadre d'interprétation naturalisant plus ou moins biologique et psychologique, en échafaudant une sorte de théorie de la perception basée sur le postulat que tous les médias constituent des extensions du corps humain : "All media are extensions of some human faculty - psychic or physical » (The Medium is the Massage, p. 26). Chaque média prolongerait l'un ou l'autre, ou plusieurs de nos cinq sens, ou notre cerveau, comme l'ordinateur.

Les médias seraient plus ou moins hot ou plus ou moins cool selon qu'ils font appel à un seul sens, transmettent une information hautement définie et impliquent une faible participation (médias hot) ou au contraire mobilisent plusieurs sens présentent une information peu précise et nécessitent un grande participation du récepteur (médias cool). Une définition relativement simple mais d'une application fort complexe dans l'œuvre de McLuhan où les médias apparaissent plus ou moins hot ou cool selon le point de comparaison. C'est ainsi, par exemple, que l'écriture alphabétique manuscrite est plus hot que l'écriture idéographique mais plus cool que l'écriture alphabétique imprimée, que la radio est parfois présentée comme cool, parfois comme hot, etc.

Les médias qui privilégient un seul sens le surchauffent et engourdissent les autres sens. La perception s'en trouve atrophiée, déséquilibrée. C'est ce qui se produit à l'ère Gutenberg. La sollicitation exagérée de la vue qu'entraîne la culture livresque a pour conséquence la linéarité, la hiérarchisation, la spécialisation et la division du savoir, la séparation entre la science et l'art, la pensée et l'action. Dans la théorie mcluhannienne, l'invention de l'imprimerie constitue la césure majeure de l'histoire.

La théorie de la perception élaborée par McLuhan, qui n'a guère de fondements scientifiques, l'a conduit à des affirmations séduisantes mais 


\section{Gaëtan TREMBLAY}

erronées comme lorsqu'il prétend que la télévision est un média tactile, radicalement différent du cinéma parce que le tube cathodique projette l'image sur la peau du téléspectateur et la tatoue alors que le projecteur cinématographique projette son image sur un écran devant le spectateur. Les théories scientifiques de la perception ne permette pas de confirmer cette assertion.

De même, quand McLuhan affirme que l'écriture est un media hot parce qu'il est une extension de la vue, le sens par excellence de la linéarité et de la segmentation dans la théorie mcluhannienne, il se fourvoie. Selon les théories scientifiques de la perception depuis la gestalt jusqu'à la simulation numérique, la vision appréhende d'abord par configuration d'ensemble, non par balayage séquentiel, et ne procède que dans un second temps à une exploration systématique de l'objet observé. Ce sont d'ailleurs les mêmes yeux qui perçoivent les images et les textes. Si l'écriture est linéaire, c'est parce qu'elle constitue un code substitutif de la chaîne parlée, non parce qu'elle s'adresse à la vue. La parole, qui sollicite l'ouie, est linéaire et non globale; elle ne se donne à entendre que dans une séquence temporelle, les signes les uns à la suite des autres. La lecture solitaire constitue bien sûr une situation de communication bien différente de la conversation en face à face. Mais il y a là bien plus qu'une opposition entre la vue et l'ouie. Ce sont les situations de communication qui sont différentes et les dimensions à prendre en considération débordent les caractéristiques purement sensorielles pour inclure des variables psychologiques, sociologiques et environnementales.

\subsection{Le vitrail, la glose et la métaphore comme instruments de travail}

Pour apprécier correctement l'œuvre de McLuhan, il faut se rappeler qu'il s'agit du travail d'un littéraire et non d'un scientifique, ni des sciences sociales ni des sciences exactes. McLuhan était un spécialiste de la littérature anglaise, plus particulièrement de celle du XVle siècle britannique. II a fait sa thèse de doctorat sur la rhétorique de Thomas Nashe, célèbre auteur satirique élisabéthain né en 1567 et mort vers 1601. II ne s'est intéressé aux médias modernes, au départ, que pour mieux comprendre ses étudiants qu'il sentait à des lieues de sa propre sensibilité.

McLuhan a grandi au sein d'une famille protestante et s'est converti au catholicisme à l'âge adulte. Je ne saurais dire dans quelle mesure sa conversion a joué un rôle majeur dans l'orientation de sa pensée. Mais il faut l'évoquer au moins sur un point, compte tenu de l'importance du dogme dans la religion catholique : le déterminisme technologique de McLuhan était incompatible avec 
De Marshall McLuhan à Harold Innis

ou du village global à l'empire mondial

sa foi, le catholicisme récusant la prédestination —voire l'idée même de destin- le sort des hommes ne dépendant que de la volonté divine et de la liberté humaine. McLuhan a donc dû trouver une façon de concilier sa pensée avec la doctrine de l'Église romaine, en particulier avec sa doctrine sociale sur les communications. II a trouvé une voie de sortie dans la formule suivante: "There is absolutely no inevitability as long as there is a willingness to contemplate what is happening " (The Medium is the Massage, p. 25). Autrement dit, les médias créent un environnement qui conditionne l'existence des hommes mais s'ils en prennent conscience, ils peuvent s'en libérer. II ne précise pas comment, tout cela restant un peu magique, mais enfin, le respect de la doctrine est sauf !

McLuhann est un littéraire. On en trouve constamment trace dans ses ouvrages, ne serait-ce que par ses nombreuses références, entre autres, à William Shakespeare et à James Joyce. Spécialiste de la Renaissance, il n'en cherche pas moins les principes de sa méthode dans la période qui l'a précédée, le Moyen Age. Pourquoi ? Pour accorder sa façon de travailler à sa théorie sur les médias. Si l'imprimerie a produit un type de raisonnement linéaire, analytique, partiel et séquentiel, qui ne saurait rendre compte de la complexité et de la globalité de l'ère Marconi, il faut trouver ailleurs les outils permettant de l'appréhender et d'en rendre compte. McLuhan les cherche et les trouve dans la pensée pré-gutenbergienne.

Se refusant à une écriture analytique qui expose de manière ordonnée une structure d'argumentation, l'oracle de Toronto procède par aphorismes et métaphores abordant les thèmes les plus divers, glose après glose, sans logique apparente d'enchaînement. Les diverses sections de ses livres peuvent se lire dans n'importe quel ordre. Pour parler de sa méthode, McLuhan aime bien faire référence à la mosaïque et au vitrail. Au point de vue systématique et linéaire de l'écriture alphabétique imprimée, il préfère le regard qui pénètre l'objet et le reconstitue par touches successives à la manière d'une mosaïque. À la lumière réfléchie qui résulte de l'éclairage extérieur de l'objet (light on), il oppose la lumière qui le traverse comme les vitraux d'une cathédrale (light through) :

Cette question s'insère dans la trame même de la pensée et de la sensibilité médiévales, tout comme elle est à la source de la pratique des "gloses », pour faire ressortir la lumière d'un texte, à la source également de la technique 


\section{Gaëtan TREMBLAY}

d'utilisation de la lumière à des effets de transparence (light through), plutôt que d'éclairage (light on), ce qui constitue le fondement même de l'architecture gothique (La Galaxie Gutenberg, p. 157).

Bref, McLuhan opte pour une approche scolastique plutôt que cartésienne. Comment, devant un tel retour aux formes de pensée d'avant la Réforme, ne pas évoquer ici encore sa conversion au catholicisme?

McLuhan n'explique pas, il explore. II aime affirmer qu'il n'a pas de point de vue, contrairement à ses contradicteurs qui justement, selon lui, s'opposent à ses vues parce qu'ils ne peuvent se départir de leur point de vue. Une position cohérente avec sa théorie des médias mais qui ne laisse aucune place au débat critique. C'est à prendre ou à laisser. Voilà peut-être ce qui explique que McLuhan ait suscité les oppositions les plus virulentes comme les adhésions les plus totales.

\section{Le scribe Innis}

Mon initiation à l'œuvre d'Harold Innis a été beaucoup plus tardive qu'à celle de Marshall McLuhan. Elle remonte à la fin des années 70 lorsque les premiers travaux devant mener à la création de l'Association canadienne des communications m'ont mis en contact régulier avec des collègues canadiens, en particulier mon amie Liora Salter, maintenant professeure à York University. L'inexistence d'une traduction française des livres d'Innis est probablement en grande partie responsable de sa faible notoriété en dehors du monde anglosaxon ${ }^{3}$.

McLuhan n'était pas un universitaire classique et il n'a jamais obtenu de ses collègues la reconnaissance à laquelle il aspirait. Innis, au contraire, représente le type même de l'intellectuel universitaire canadien. On le disait piètre pédagogue mais un érudit et un chercheur respecté. Bien après sa mort, il est resté un modèle de référence pour plusieurs universitaires canadiens. À bien des égards, Innis incarnait un représentant typique de la culture livresque de l'ère Gutenberg, selon McLuhan. La figure qu'évoque son personnage est davantage celle du scribe studieux que celle du prophète charismatique.

Sans doute la formation en sciences sociales de Harold Innis m'a-t-elle d'emblée rendu son approche des communications plus crédible que celle de

3 Le seul texte d'Innis publié en français est «L'oiseau de Minerve », Communication Information, Québec, 1983, vol. V, nos 2 et 3, p. 267-297, traduction de Roger de la Garde et Line Ross.

tic\&société - 1(1), 2007 
De Marshall McLuhan à Harold Innis

ou du village global à l'empire mondial

McLuhan. Je suis d'abord un sociologue, fasciné par l'histoire et l'économie. Innis était avant tout un économiste, passionné d'histoire. Ses premiers travaux ont porté sur les économies fondées sur l'exploitation et l'exportation des matières premières, en particulier la fourrure et le bois canadiens. Le constat du rôle central des infrastructures de transport l'a conduit peu à peu à l'étude des communications.

\subsection{Les médias, instruments de contrôle du temps et de l'espace}

Innis est l'un des premiers chercheurs à avoir mis en évidence l'importance stratégique des communications dans la création et la survie des empires depuis les tout débuts de la civilisation. La théorie innissienne s'articule en fonction des concepts de base que sont le temps et l'espace. Toute société doit s'aménager un territoire et se donner les moyens de survivre et de se reproduire. Selon Innis, tous les médias présentent des biais ${ }^{4}$, des caractéristiques qui permettent de contrôler l'une ou l'autre dimension, l'espace ou le temps. Tout empire, toute société qui prétend à une certaine pérennité doit réaliser un équilibre entre un média qui favorise le contrôle de l'espace et un autre qui assure sa reproduction dans le temps. Enfin, selon Innis, le fonctionnement de ces médias dominants suscite la création de monopoles du savoir, exercé par une caste ou un groupe de prêtres, de scribes, de savants à qui le pouvoir octroie un certain nombre de privilèges.

On ne trouve trace d'aucune théorie de la perception dans l'œuvre d'Harold Innis. Les médias n'y sont pas conçus comme des extensions des organes des sens humains. Les biais qu'ils impliquent, les caractéristiques qu'ils présentent dépendent de la nature de leur support (lourd ou léger, durable ou éphémère, etc.), de leur plus ou moins grande facilité d'accès (rareté des lieux de production du papyrus, décentralisation de la production du papier, etc.), de leurs conditions de production (lenteur de la sculpture sur pierre; rapidité d'exécution des signes sur l'argile qui sèche vite; proximité et abondance de réserves d'eau pour la production du parchemin; etc.) et du savoir nécessaire à cette production.

4 Le mot biais traduit bien imparfaitement le sens du terme anglais bias impliquant l'idée, chez Innis, que les médias possèdent certaines caractéristiques qui en orientent et conditionnent les usages et les effets. 


\section{Gaëtan TREMBLAY}

Certains médias, par leur légèreté, leur facilité de production et de transport, se révèlent des instruments utiles, efficaces, voire indispensables au contrôle politique et administratif qu'implique le fonctionnement d'un empire dominant un vaste territoire. C'est le cas du papyrus et du papier, par exemple. D'autres, à l'opposé, présentent des traits qui les rendent mieux adaptés à la transmission dans le temps. Le monopole en a été historiquement assumé par le pouvoir religieux. Leur production est longue et difficile mais leur durée de vie se mesure en siècles et en millénaires. C'est le cas de l'écriture gravée sur la pierre.

\subsection{Communication et société}

Armé de ces quelques concepts (temps, espace, biais, équilibre, monopole du savoir), Innis s'est livré à une étude minutieuse des documents historiques pour tenter de valider ses hypothèses. Les résultats en sont exposés dans ses deux ouvrages sur les communications, The Bias of Communication et Empire and Communication. S'en détachent quelques grands empires comme celui de l'ancienne Egypte, fondée sur le double usage de la pierre et du papyrus; celui de la Grèce du siècle de Périclès, heureux mélange de tradition orale et de civilisation écrite; celui de Byzance, qui a combiné les avantages du papyrus et du parchemin.

Si McLuhan était obsédé par le changement consécutif à l'invention de nouveaux médias, Innis se montre surtout préoccupé d'équilibre et de stabilité. Son analyse fait bien sûr place au changement, lequel prend naissance en périphérie de l'empire, du territoire soumis à l'emprise des médias dominants. Mais il cherche surtout à établir les conditions de contrôle et de survie spatiotemporelles des sociétés :

It has seemed to me that the subject of communication offers possibilities in that it occupies a crucial position in the organization and administration of government and in turn of empires and of Western civilization (Empire and Communication, p. 5) ${ }^{5}$.

La pensée d'Innis est entièrement dominée par le postulat de l'équilibre, cher à nombre d'économistes. Les modèles de sociétés qu'il a le plus valorisés sont ceux qui ont atteint une stabilité qui leur a permis de traverser les siècles. Innis a surtout admiré les sociétés pré-industrielles, les sociétés du passé où les médias étaient peu nombreux et le rythme du changement était plutôt lent. II a

5 « II m'est apparu que le sujet de la communication recèle un riche potentiel du fait qu'il occupe une place centrale dans l'organisation et l'administration du gouvernement, des empires et de la civilisation occidentale »(ma traduction).

tic\&société - 1(1), 2007 
De Marshall McLuhan à Harold Innis

ou du village global à l'empire mondial

écrit dans la première moitié du XXe siècle, ne l'oublions pas. II a peu connu la télévision, encore moins l'ordinateur et pas du tout les réseaux numériques. On peut à juste raison se demander si ses hypothèses font toujours sens, si elles peuvent être transposées à l'étude des sociétés modernes où cohabitent une pléthore de médias et où le changement est la seule valeur stable. L'équilibre est-il possible dans ces sociétés ? Est-il nécessaire ?

Par ailleurs, peut-on encore catégoriser les médias modernes selon qu'ils présentent un biais spatial ou temporel ? McLuhan a raison, l'électricité a changé complètement la donne. Et comme le fait remarquer James Carey, depuis l'invention du télégraphe électrique le rapport entre les communications et le transport s'est totalement inversé. Non seulement la transmission des messages s'est-elle autonomisée des moyens de transports physiques des marchandises, c'est elle qui permet maintenant de réguler la circulation des biens matériels et des personnes. Historiquement dépendante des moyens de transports, la communication s'en est affranchie avec l'électricité et la gestion des transports ne sauraient maintenant se passer des moyens modernes de communication.

Les médias électriques et électroniques autorisent certes un contrôle de l'espace comme jamais auparavant dans l'histoire. Mais doit-on en conclure qu'ils sont inadaptés à la conservation et à la transmission dans la longue durée ? La plupart des messages sont éphémères mais cette courte existence dépend davantage de leur utilité relative que des caractéristiques des médias. Les inscriptions numériques sur disques ne peuvent-elles pas traverser les siècles tout autant que les bas-reliefs gravés dans la pierre?

Malgré ces réserves sur la pertinence des hypothèses innissiennes sur l'équilibre des médias pour l'analyse des sociétés contemporaines, les travaux de James Carey ont bien montré tout le profit qu'on peut tirer de l'application d'un cadre conceptuel s'inspirant de l'approche d'Innis.

\subsection{La méthode innissienne}

La méthode d'Harold Innis n'a rien à voir avec celle d'un Marshall McLuhan. Innis, rappelons-le, a été formé à l'école des sciences sociales. Sa méthode de travail repose sur l'étude minutieuse des livres d'histoire, l'accumulation d'indices et de preuves, le croisement des sources d'information. S'il fait inévitablement usage de métaphores comme tout auteur, il se garde bien de l'ériger en méthode d'exploration. Et si la lecture de ses textes est souvent 


\section{Gaëtan TREMBLAY}

laborieuse, c'est en grande partie à cause de l'érudition nécessaire à leur compréhension.

En plaçant les techniques de communication au cœur de l'évolution historique, la pensée d'Innis peut certes être qualifiée de déterministe. Le déterminisme technologique sous-jacent au modèle de la société de l'information, dans lequel l'évolution des technologies de l'information et de la communication est présentée comme le facteur déterminant du changement, en fait nécessairement une explication réductrice incapable de prendre en considération les stratégies d'acteurs et les conflits qui en résultent. C'est également une lecture qui fait l'impasse sur tous les problèmes et les défis qui confrontent les sociétés contemporaines mais qui ne ressortissent pas directement ou principalement au développement des techniques de communication et d'information : les inégalités sociales, la pauvreté, la marginalité et l'exclusion, etc.

Une théorie de la communication qui a pour objectif de rendre compte du social doit nécessairement être articulée à une théorie de la production de biens matériels (de transformation du monde, de production de richesses) et à une théorie des relations de pouvoir, bref s'inscrire dans une économie politique de la communication. Cet impératif s'impose d'autant plus dans nos sociétés contemporaines où la communication, depuis la création de ses produits jusqu'à leur distribution, est largement et profondément intégrée au mode de production capitalistique.

L'œuvre d'Harold Innis constitue un bel exemple de ce nécessaire arrimage des sciences de la communication aux autres sciences sociales, tout particulièrement l'histoire, la sociologie, l'économique et la science politique. Si la thèse d'Innis est construite autour du rôle central que jouent les techniques de communication dans la constitution et la survie des empires, les concepts de monopole ou d'oligopole du savoir et celui d'équilibre entre les médias à biais temporel et à biais spatial - transposition de concepts empruntés à la science économique - remplissent une fonction essentielle dans la construction de son argumentation. Et si le point de départ de sa pensée comporte un a priori en faveur d'un certain déterminisme technologique, l'analyse historique, fine et détaillée à laquelle il se livre accorde une importance capitale aux notions d'intérêt, de pouvoir, d'accessibilité et de répartition des ressources. II en résulte un modèle d'explication beaucoup plus complexe que ce que pouvait laisser entrevoir l'hypothèse de départ. De plus, Innis rappelle d'entrée de jeu le statut d'hypothèses de ses assertions et met en garde le lecteur contre ses éventuels biais personnels : 
De Marshall McLuhan à Harold Innis

ou du village global à l'empire mondial

But I must confess at this point a bias which has led me to give particular attention to this subject. In studies of Canadian economic history or of the economic history of the French, British, and American empires, I have been influenced by a phenomenon strikingly evident in Canada, which for that reason I have perhaps overemphasized (Empire and Communication, p. 5) ${ }^{6}$.

In any case I have tried to present my bias in order that you may be on your guard (Empire and Communication, p. 6) ${ }^{7}$.

The generalizations which we have just noted must be modified in relation to particular empires (Empire and Communication, p. 11) ${ }^{8}$.

Voilà une attitude qui tranche avec celle d'un McLuhan qui se veut à l'abri des points de vue!

\section{L'héritage d'Innis et de McLuhan}

Les travaux d'Innis et de McLuhan sont-ils dépassés ou toujours d'actualité ? Dans quelle mesure peuvent-ils encore servir à l'étude des phénomènes contemporains de communication ? Que faut-il retenir de l'un et de l'autre ? J'ai déjà au passage formulé quelques remarques critiques sur leurs postulats, leurs hypothèses, leurs concepts ou leurs méthodes. Je n'y reviendrai pas en détails mais je voudrais souligner que leur contribution à l'étude des phénomènes de communications m'apparaît fondamentale sous au moins trois aspects. Je voudrais par la suite soumettre à la discussion critique la plus ou moins grande

6 « Je dois confesser ici un bias qui m'a conduit à prêter une attention particulière à ce sujet. Dans les études sur l'histoire économique canadienne ou sur l'histoire économique des empires français, anglais et américain, j'ai été influencé par un phénomène d'une évidence patente au Canada, que pour cette raison j'ai peut-être surestimé "(ma traduction).

7 «Dans tous les cas, j'ai essayé d'exposer mon bias pour que vous restiez sur vos gardes $\gg$ (ma traduction).

8 "Les généralisations que nous venons de signaler doivent être modulées en fonction des empires spécifiques »(ma traduction). 


\section{Gaëtan TREMBLAY}

actualité de leurs pronostics concernant le village global et le destin des empires.

\subsection{Les apports à la recherche sur les médias}

Innis et McLuhan ont, chacun à leur manière, mis en évidence le rôle structurant des communications dans l'organisation des sociétés comme dans la vie des individus. C'est dorénavant un constat universellement reconnu, un acquis indéniable. L'on ne peut comprendre et expliquer l'évolution des sociétés modernes et post-modernes sans prendre en compte les mutations des secteurs de l'information et de la communication. Nul ne conteste maintenant que les techniques de communication jouent un rôle essentiel dans l'organisation économique, politique, culturelle et sociale des collectivités humaines.

Cet acquis comporte cependant souvent un corollaire encombrant : le déterminisme technologique. Malgré les dénégations des uns et des autres, c'est un postulat trop largement partagé dans les études en communications. Manuel Castells en fournit un exemple éloquent dans sa trilogie sur l'ère de l'information. Il évoque trois facteurs principaux pour expliquer les changements structurels dans les relations économiques, sociales et politiques qu'ont connu les sociétés occidentales entre la fin des années 1960 et le milieu des années 1970 : 1) la révolution des techniques de l'information ; 2) la crise du capitalisme et de l'étatisme ; 3) l'effervescence des mouvements sociaux comme l'environnementalisme et le féminisme. Mais entre les trois, selon lui, c'est la révolution des techniques de communication qui a joué le rôle déterminant (Tremblay, 2003).

II est à souhaiter que les chercheurs en communication qui s'attaquent à la difficile tâche de fournir une vue d'ensemble et un modèle d'explication global des mutations qui affectent les sociétés dans lesquelles nous vivons adoptent une approche qui rende davantage compte de la complexité des facteurs en cause.

Les contributions d'Innis et de McLuhan aux sciences de la communication m'apparaissent décisives sous un autre rapport. Dans le secteur des communications comme en d'autres domaines, les changements les plus profonds qui accompagnent les innovations techniques ne peuvent être correctement appréhendés qu'à long terme. Les recherches empiriques à brève échéance, si sophistiquées et si élaborées soient-elles, constituent des instruments bien limités pour identifier les tendances de fond. 
De Marshall McLuhan à Harold Innis

ou du village global à l'empire mondial

Restent trois voies possibles en sciences sociales, qui présentent chacune leur utilité et leurs inconvénients.: les études longitudinales, les travaux historiques, les méthodes prospectivistes. Les études longitudinales peuvent être définies et contrôlées en fonction d'objectifs précis mais elles sont fort coûteuses. Les travaux historiques sont plus abordables mais on ne peut jamais présumer que leurs résultats sont transposables au présent et au futur. Les études prospectivistes permettent d'explorer l'univers des possibles mais leur fiabilité est douteuse, l'expérience ayant démontré que le taux de succès des pronostics -y compris ceux formulés par les plus grands spécialistesdépasse rarement celui d'une performance aléatoire.

L'œuvre d'Innis et celle de McLuhan montrent l'intérêt de recourir aux approches historique et prospectiviste dans la mesure où l'on en cerne bien les limites et qu'on se garde de conclure que les leçons du passé peuvent être projetées dans l'avenir et que les projections futuristes ont valeur d'observations scientifiques. Elles en illustrent aussi les dérives auxquelles elles peuvent conduire si l'on fait fi de ces précautions.

Les contributions d'Innis et McLuhan à l'étude des communications me semblent significatives sous un troisième aspect. En analysant l'impact des médias eux-mêmes plutôt que l'effet des messages qu'ils véhiculent, ils ont introduit dans les recherches sur les médias de masse, comme on disait après la seconde Guerre mondiale, la matérialité de l'objet, dans ses caractéristiques techniques, ses conditions de production et d'utilisation, son insertion dans l'espace et dans le temps. On ne trouvera ni chez l'un ni chez l'autre les fondements d'une théorie de l'innovation ou d'une théorie des usages sociaux des technologies d'information et de communication. Le diffusionnisme d'un Everett Rogers, la théorie de la traduction de Callon et Latour ou les théories contemporaines de l'appropriation ou encore celle des communications pour le développement ne sont pas les héritières directes des travaux des deux chercheurs canadiens. Mais depuis leurs publications des années 50 et 60 , il n'est plus possible de considérer les médias comme des instruments totalement neutres et plastiques pouvant se prêter à toutes fins et en toutes circonstances. Même sans partager le postulat du déterminisme technologique, on ne peut plus penser les médias uniquement comme de simples moyens en vue de quelque chose d'autre. Les travaux d'Innis et de McLuhan ont établi de façon définitive que chaque média présente une configuration spécifique de possibilités et de contraintes que toute théorie des communications doit prendre en compte. 


\section{Gaëtan TREMBLAY}

\subsection{Du village global à l'empire mondial}

McLuhan pensait que les nouvelles technologies d'information et de communication transformeraient le monde en un gros village. Innis en venait lui à la conclusion que le déséquilibre en faveur des médias à biais spatial causé par les technologies modernes rendrait impossible la création de nouveaux empires pérennes. Qu'en est-il de ces pronostics?

Dans la Galaxie Gutenberg, publié il y a plus de quarante ans, McLuhan annonçait déjà l'avènement du village-monde :

Cette situation [celle d'une société orale où
l'interdépendance résulte de l'interaction nécessaire
des causes et des effets dans la totalité de la
structure] est typique d'un village et, depuis
l'avènement des moyens électroniques de
communication, du village global. Aussi est-ce le
monde de la publicité et des relations publiques qui est
le plus conscient de cette dimension nouvelle et
fondamentale qu'est l'interdépendance globale (La
Galaxie Gutenberg, p. 38).

Et il l'a répété avec force par la suite :

The new electronic interdependence recreates the world at the image of a global village (The Medium is the Massage, p. 67) ${ }^{9}$.

On donnera volontiers raison à McLuhan sur l'interdépendance croissante du monde qui résulte de l'amélioration des transports et de l'accroissement des échanges économiques tout autant que de l'expansion des réseaux de communication, enclenchés dès le Quattrocento avec les grandes découvertes et la montée en puissance du capitalisme commercial. Mais la métaphore du village, fort populaire puisqu'elle a fait le tour de la planète et traversé quatre décennies, est-elle éclairante pour comprendre le processus de globalisation ou de mondialisation ? Après mûre réflexion, la réponse est « non » et il me semble qu'il faut chercher ailleurs que dans la justesse de l'image, ailleurs que dans sa capacité de représentation, les raisons de son succès. J'y reviendrai.

La métaphore du village n'est pas adéquate pour évoquer l'interdépendance croissante du monde pour au moins deux bonnes raisons. Premièrement, l'image du village rend mal compte du processus en cours parce que les

9 «La nouvelle interdépendance électronique recrée le monde à l'image d'un village global » (ma traduction).

tic\&société - 1(1), 2007 
De Marshall McLuhan à Harold Innis

ou du village global à l'empire mondial

réseaux d'échanges et de communication relient davantage les villes que les villages. La globalisation est avant tout l'affaire des grandes villes de la planète. Les habitants des villages et des campagnes y sont soit faiblement intégrés, soit tenus à l'écart.

La deuxième raison, plus fondamentale encore, pour laquelle la métaphore du village me semble inadéquate, c'est parce qu'elle laisse entendre que l'interdépendance y serait plus grande que dans une ville. C'est plutôt le contraire qui est vrai. L'on sait en effet depuis les premiers travaux des pionniers de la sociologie et de l'économie politique que plus la division du travail est élaborée dans une collectivité plus grande y est l'interdépendance entre ses membres. Or, point n'est besoin d'argumenter longtemps pour démontrer que la division du travail est infiniment plus complexe dans une grande ville que dans un village. Le citadin est beaucoup plus dépendant de ses semblables que le villageois. II ne les connaît pas personnellement, contrairement au villageois qui peut nommer pratiquement tous les habitants de son bled par leur nom. Mais c'est là une autre affaire.

En fait, si la métaphore du village global est si populaire, c'est, me semble-til, parce qu'elle connote à d'autres signifiés que les liens réels d'interdépendance qui se développent dans le monde moderne. Le village, pour les citadins - parmi lesquels se recrutent la plus grande partie des lecteurs de McLuhan et des adeptes du village global- fait référence au stéréotype de l'endroit calme et agréable situé dans un environnement idyllique, sans bruit ni pollution, où vivent dans l'harmonie, l'amour et l'amitié les membres d'une petite communauté solidaire. Le mythe du village présente les traits sublimés de ce que Tönnies a défini comme une communauté, par opposition à une société. La métaphore du village global marche parce qu'elle exprime l'espoir insensé que l'avenir nous conduise à la reproduction d'un passé idéalisé. Le problème, c'est que ce n'est pas du tout ce type d'interdépendance qui résulte de l'expansion des réseaux d'échange et de communication.

Nous sommes beaucoup plus près de la réalité avec cet autre aphorisme de McLuhan, même si l'on y trouve inévitablement trace de son déterminisme technologique ${ }^{10}$ :

10 Une approche moins déterministe ferait ressortir le rôle majeur de l'armée dans la recherche technique et le poids qu'exerce, dans le déclenchement des conflits, son désir de mettre à l'épreuve ses nouvelles armes. Ce n'est pas la technologie en soi qui a 


\section{Gaëtan TREMBLAY}

Every new technology necessitates a new war (War and Peace in the Global Village, p. 98) ${ }^{11}$.

On pense tout de suite évidemment à la Guerre du Golfe en 1990 et à celle d'Iraq en 2003 qui auront permis aux Américains de tester leurs armes "intelligentes" et de faire la démonstration de leur considérable avance technologique sur tous les autres pays de la planète. Peut-on parler d'un empire américain, comme nous y invitent Negri et Hardt?

La question mérite certainement d'être posée et débattue. Cet empire dont l'écrasante supériorité militaire lui permet de s'affirmer comme seule superpuissance du monde a-t-il des chances de durer ou est-il déjà sur son déclin, comme le pense en particulier le cinéaste Denys Arcand, dont le plus récent film Les invasions barbares - en quelque sorte la suite d'un film précédent, intitulé Le déclin de l'empire américain, tourné il y a une vingtaine d'années - lui a mérité une palme pour le meilleur scénario au dernier festival de Cannes (2003) ?

Harold Innis partagerait sans doute le diagnostic de Denys Arcand. Selon sa théorie, les Américains possèdent incontestablement la technologie adéquate pour contrôler de vastes territoires distants les uns des autres, mais on peut se demander s'ils peuvent compter sur un média capable d'assurer la pérennité des valeurs sur lesquelles se fonde le système américain. L'empire américain finira un jour par tomber, comme tous les précédents, mais quand ? Seule l'histoire, évidemment, apportera réponse à ces interrogations. Les scénarios apocalyptiques sur l'hégémonie américaine ne me semblent cependant pas plus convaincants que ceux qui prédisaient la mort du système capitaliste dans les années 60 ou la fin de l'histoire dans les années 90 . Les Américains ne croient sans doute plus avec la même ferveur au rêve de régénération qui a animé leurs ancêtres mais les valeurs de base du libéralisme, sur lesquelles repose le fonctionnement de leur système économique et social, se transmettent encore d'une génération à l'autre. De plus, l'importance du fait religieux aux États-Unis ne doit pas être sous-estimée. Ne doit-on pas même y craindre la montée du fondamentalisme?

Mais laissons ces supputations futurologiques et revenons à l'exercice bien réel de l'hégémonie américaine contemporaine. James Carey a écrit que l'invention du télégraphe électrique avait permis le passage du colonialisme,

besoin de la guerre mais les groupes sociaux qui la mettent au service de leur attitude belliqueuse.

11 «Chaque nouvelle technologie appelle une nouvelle guerre » (ma traduction).

tic\&société - 1(1), 2007 
De Marshall McLuhan à Harold Innis

ou du village global à l'empire mondial

mode de domination décentralisé dans lequel la lenteur des transports et des communications impliquait forcément une certaine autonomie du gouverneur local, à l'impérialisme moderne à l'intérieur duquel la rapidité des communications rend quotidienne l'influence du pouvoir central. L'informatique et les réseaux télématiques ont renforcé considérablement cette tendance, à un point tel qu'on peut se demander si nous n'assistons pas à l'émergence d'une nouvelle forme d'impérialisme, qui pourrait, dans la plupart des cas, faire l'économie de la mise en place d'une administration politique tentaculaire à la charge du pouvoir central. Un nouvel empire fondé sur l'exercice du pouvoir en réseau, comme le qualifient Hardt et Negri (2000) ?

La notion d'impérialisme culturel, popularisée par Herbert Schiller dans les années 70 , a été sévèrement critiquée pour reposer, exagérément affirmait-on, sur une théorie du complot et de la manipulation. On lui a opposé, comme pour en banaliser l'importance, la liberté et l'autonomie des récepteurs. En ces temps où l'on parle tant de globalisation et de mondialisation, il ne serait pourtant pas inutile de ré-évaluer les hypothèses de Schiller, et de les interroger, en particulier, sous l'angle des préoccupations innissiennes concernant la transmission et la pérennité des valeurs. Un politologue et conseiller de la Maison blanche, Joseph Nye, n'a-t-il pas sérieusement proposé, en 1990, une stratégie de soft power qui intègre en bonne partie ce que Schiller entendait par impérialisme culturel (Mattelart et Tremblay, 2003) ?

II semble cependant que l'actuelle administration américaine ait choisi une autre voie, plus brutale, comme on a pu le voir lors de l'intervention d'avril dernier 2003 en Iraq. Faut-il y voir un changement de cap ou seulement une nouvelle phase dans une stratégie globale qui manie alternativement la carotte et le bâton ? L'hégémonie culturelle constitue-t-elle réellement une alternative à l'exercice de la force armée ou les deux ne sont-ils que deux facettes d'une même politique?

\section{Conclusion}

J'ai seulement rappelé, à grands traits, la pensée et l'approche des deux pionniers de la recherche en communication au Canada. J'ai soulevé quelques questions pour relativiser certaines de leurs conclusions et poursuivre la réflexion. Mais, pour comprendre l'œuvre d'Innis et celle de McLuhan, il resterait encore à les resituer dans le contexte socio-économique de leur élaboration, dans cette période de l'après Deuxième Guerre mondiale, si fertile en 


\section{Gaëtan TREMBLAY}

changements majeurs dans tous les domaines que je n'essaierai même pas de les évoquer succinctement. Je mentionnerai seulement l'avènement, dans les années 50 , de la télévision, devenue le plus populaire et le plus puissant des médias de communication; et la création de I'UNESCO, à titre d'indice de la reconnaissance nouvelle, à l'échelle planétaire, du rôle de la culture, de l'éducation et de la communication dans la vie des sociétés et le développement des peuples.

II faudrait aussi, pour apprécier la contribution intellectuelle d'Innis et celle de McLuhan, mettre leurs travaux en perspective avec ceux d'autres auteurs de la même époque en d'autres coins du monde. Au moment où les deux auteurs canadiens mettaient la technique au centre de leur étude des communications, la plupart des chercheurs américains poursuivaient leurs recherches empiriques sur les effets des messages. D'autres appliquaient les thèses évolutionnistes et diffusionnistes aux communications pour le développement. Adorno et Horkheimer critiquaient l'industrie culturelle tandis que Shannon et Weaver mettaient au point la théorie mathématique de l'information et que Wiener jetait les bases de la cybernétique. Les Européens de l'Ouest, en particulier les Français, influencés par le structuralisme en linguistique et en anthropologie, travaillaient sur le sens et faisaient de la sémiologie LA science des communications. Au Royaume-Uni, les travaux de Hoggart, de Williams et de Thompson sur la culture populaire et la formation des adultes, inspirés au départ par les approches de la philosophie marxienne, devaient conduire à la création du puissant courant, devenu protéiforme, des Cultural Studies.

II faudrait enfin, pour bien saisir l'impact des œuvres d'Innis et de McLuhan, se demander pourquoi des théories au fondement empirique fragile, si peu orientées vers l'action, si peu traduisibles en démarches concrètes, ont-elles connu un si grand succès auprès des gestionnaires publics et privés. Pourquoi le déterminisme technologique fait-il tant recette auprès des décideurs, des technocrates et des administrateurs en tout genre?

\section{Références Bibliographiques}

ADORNO T.W., 1964, "L'industrie culturelle », Communications (3), pp.12-18.

ADORNO T.W., M. HORKHEIMER, 1974, «La production industrielle de biens culturels », La raison dialectique. Paris, Gallimard (1ère édition: 1947).

CAREY J. W., 1989, Communication as Culture. Essays on Media and Society, Boston, Unwin Hyman.

CASTELLS M., 1998, La société en réseaux. L'ère de l'information, Paris:

Fayard, (édition en anglais:1996) 
De Marshall McLuhan à Harold Innis ou du village global à l'empire mondial

CASTELLS M., 1999, Le pouvoir de l'identité. L'ère de l'information II, Paris: Fayard, (édition en anglais:1997)

CASTELLS M., 1999, Fin de millénaire. L'ère de l'information III, Paris: Fayard, (édition en anglais:1998)

CASTELLS M., 2001, La Galaxie Internet, Paris, Fayard.

CHRISTIAN W. (Introduced and edited by), 1980, The Idea File of Harold Adams Innis, Toronto, The University of Toronto Press

FERANDEZ COLLADO C. y HERNANDEZ SAMPIERI R. , 1995, Marshall McLuhan, el explorador solitario, México, Grijalbo, Universidad Iberoamericana.

HARDT M. and NEGRI, A.,2000, Empire, Paris, Exils Éditeur.

INNIS H. A., 1972, Empire and Communications, Toronto, University of Toronto Press (1ère édition : 1950).

INNIS H. A., 1977, The Bias of Communication, Toronto, University of Toronto Press (1ère édition: 1951).

MARCUSE H., 1969, Vers la libération. Au-delà de l'homme unidimensionnel, Paris, Éditions de Minuit , 1979.

MATTELART A. et G. TREMBLAY, 2003, "Communication et démocratie ", Actes du colloque 2001 Bogues, globalisme et pluralisme, Québec, Presses de l'Université Laval.

MCLUHAN M., 1951, The Mechanical Bride, Boston, Beacon Press, 4th printing, 1969.

MCLUHAN M. and FIORE Q., 1968, War and Peace in the Global Village, New York, Bantam Books.

MCLUHAN M. and FIORE Q., 1967, The Medium is the Massage. An Inventory of Effects, Bantam Books.

MCLUHAN M., 1970, Pour comprendre les média ${ }_{1} \mathrm{HMH}$, Montréal, (édition originale en anglais publiée par New York, McGraw-Hill, 1964)

MCLUHAN M., 1968, La galaxie Gutenberg, HMH, Montréal, (édition originale en anglais publiée par Toronto University Press, 1962) 


\section{Gaëtan TREMBLAY}

MELODY W., SALTER, L., HEYER, P. (editors), 1981, Culture, Communication and Dependency, The tradition of H. A. Innis, Norwood, New Jersey, Ablex Publications Corporation

NYE J., 1990, Bound to Lead: The Changing Nature of American Power, New York, Basic Books.

SALTER L., ed., 1981, Communication Studies in Canada, Toronto, Butterworths.

SCHILLER H., 1976, Communication and Cultural Domination, White Plains, NY, M. E. Sharpe

TONNIES, F., 1922, Communauté et société (Titre allemand original: Gemeinschaft und Gesellschaft), Paris, Les Presses universitaires de France, 1977, Collection: Les classiques des sciences humaines.

TREMBLAY G., 1995, « The Information Society: From Fordism to Gatesism », Canadian Journal of Communication, vol. 20, no 4, pp. 461-482. Reprinted in "Regulationist Perspectives on Fordism and Post-Fordism, Regulation Theory and the Crisis of Capitalism," Bob Jessop editor, Edward Elgar Publisher, Cheltenham, UK - Northampton, MA, USA, 2001, pp. 392-413. En français : "La société de l'information : du fordisme au gatesisme ", Communication Information, vol. 16, no 2, décembre 1995, pp. 131-158.

TREMBLAY G., 2002, « Le développement de la recherche en communication. Éléments pour une analyse comparée Brésil-Canada », Colloque Amérique. Terre d'utopies. Les défis de la communication sociale, Salvador de Bahia, Brésil, $1^{\text {er }}$ et 2 septembre 2002 <http://www.er.uqam.ca/nobel/gricis/even/Utopie/francais/u_pr_f.html>

TREMBLAY G., 2003, « La Sociedad de la Información y la nueva economía. Promesas, realidades y faltas de un modelo ideológico », TÉLOS, Madrid, No 54, p. 16-23.

\section{Pour citer cet article :}

TREMBLAY G., «De Marshall McLuhan à Harold Innis», Revue tic\&société [En ligne], Volume 1, n9, 2007, mis en ligne le : mis à jour le : 15/02/2008, URL :

<http://revues.mshparisnord.org/lodel/ticsociete/index.php?id=222> 